\title{
Civilisations
}

Revue internationale d'anthropologie et de sciences

humaines

$41 \mid 1993$

Mélanges Pierre Salmon II

\section{Tippo Tip à Mulongo. Nouvelles données sur le début de la pénétration arabo-swahili au Sahara}

Pierre de Maret et Hugues Legros

\section{OpenEdition \\ Journals}

Édition électronique

URL : http://journals.openedition.org/civilisations/1725

DOI : 10.4000/civilisations. 1725

ISSN : 2032-0442

Éditeur

Institut de sociologie de l'Université Libre de Bruxelles

\section{Édition imprimée}

Date de publication : 1 septembre 1993

Pagination : 377-401

ISBN : 2-87263-094-5

ISSN : 0009-8140

\section{Référence électronique}

Pierre de Maret et Hugues Legros, «Tippo Tip à Mulongo. Nouvelles données sur le début de la pénétration arabo-swahili au Sahara », Civilisations [En ligne], 41 | 1993, mis en ligne le 30 juillet 2009, consulté le 19 avril 2019. URL : http://journals.openedition.org/civilisations/1725 ; DOI : 10.4000/ civilisations. 1725

Ce document a été généré automatiquement le 19 avril 2019.

(c) Tous droits réservés 


\title{
Tippo Tip à Mulongo. Nouvelles données sur le début de la pénétration arabo- swahili au Sahara
}

\author{
Pierre de Maret et Hugues Legros
}

$1 \mathrm{Au} 18^{\circ}$ et $19^{\circ}$ siècles, l'empire luba fut l'un des plus grand états dynastiques de l'Afrique Centrale. Ses dirigeants réclamaient tribut sur un territoire s'étendant de la frange sud de la forêt équatoriale zaïroise jusqu'au nord de la Zambie. La réputation et le prestige de cet empire étaient tels que de nombreux autres états de la région - que ce soit les Lunda ou les Bemba par exemple -, se réclament encore d'un ancêtre Luba prestigieux ${ }^{1}$.

2 Resté à l'écart du commerce à longue distance qui, au cours du $19^{\circ}$ siècle, se déroulera plus au sud, par l'intermédiaire des Lunda et des Bemba, l'état luba ne s'ouvrira que fort tard aux influences extérieures. Les débuts de la pénétration étrangère dans le pays luba ne datent d'ailleurs que du dernier tiers du $19^{\circ}$ siècle, quand, sous l'impact du commerce à longue distance qui s'établit avec l'Angola à l'ouest et la Tanzanie à l'est, on assistera à l'effondrement de cet état.

3 Ce contexte remarquable rend l'étude du début de la pénétration étrangère dans cette région particulièrement importante, d'autant plus qu'un des premiers commerçant arabe à atteindre le coeur de l'empire luba n'est autre que le célèbre Hamed ben Mohammed el Murjebi, surnommé Tippo Tip. Dans sa Maisha, son autobiographie rédigée en Swahili, publiée et traduite en allemand par Brode en 1902, Tippo Tip relate ses incursions en pays luba, de manière malheureusement assez brève. Jusqu'à ce jour, aucune autre source ne permettait de vérifier les informations fournies par Tippo Tip, ni de les compléter.

En 1975, l'un d'entre nous a eu la chance de découvrir chez un notable de la chefferie luba de Mulongo, un manuscrit sur papier pelure qui était la copie, datée de 1935, d'un rapport administratif établi en 1909 par l'administrateur de territoire Boterdal. Ce document inédit, rédigé à partir des traditions orales du siècle précédent, se rapporte aux luttes que se livrèrent pour le pouvoir deux lignages royaux rivaux de la chefferie de Mulongo. Il nous fournit non seulement une généalogie complète des chefs, mais permet aussi de 
préciser et de développer l'historique de la pénétration arabe, yeke et européenne dans le royaume luba. Par ailleurs, ce document fait aussi référence au passage de Tippo Tip dans la chefferie de Mulongo et nous renseigne amplement sur les événements survenus durant ce séjour.

Ainsi, deux sources, toutes deux orales à la base et retranscrites au début du siècle, relatent le passage de Tippo Tip à Mulongo et le début de la pénétration arabe en pays luba. Pour un historien des traditions orales, il s'agit là d'un corpus tout à fait exceptionnel, puisque nous nous trouvons en présence de deux sources orales dont on peut prouver l'indépendance de l'une par rapport à l'autre. En règle générale, lorsque l'on est confronté à plusieurs traditions orales se référant à des événements similaires, on peut supposer qu'elles sont dépendantes l'une de l'autre, qu'elles proviennent, à l'origine, d'une tradition commune ${ }^{2}$. Or, dans le cas qui nous préoccupe, nous avons la quasi certitude que ces deux sources sont totalement indépendantes l'une de l'autre. En effet, d'un côté, il est impossible que Tippo Tip, lorsqu'il relate ses souvenirs au début du siècle, ait pu prendre connaissance d'un rapport administratif du Congo Belge et que, d'autre part, Boterdal n'a pu posséder un exemplaire des mémoires de Tippo Tip puisque la première édition, datant de 1902, n'aurait pu parvenir au coeur du Katanga en 1909, date de la confection du rapport de Boterdal. De plus, ce texte capital pour l'histoire de l'Afrique Centrale ne sera que peu utilisé par les historiens. Il faudra attendre 1959 pour qu'un ouvrage en français ait recours aux mémoires du célèbre commerçant (P. Ceulemans, La question arabe et le Congo, Bruxelles, 1959)3. Habituellement, comme l'écrit J. Vansina, "Chronology and lack of independence are real problems for oral traditions. They can be overcome or alleviated in some cases by outside evidence, but, because the contents of outside evidence tend not to be congruent with the contents of oral tradition, such cases will remain the exception rather than the rule" ${ }^{4}$. Cependant, dans ce cas-ci, la parfaite congruence des sources orales - que nous savons par ailleurs indépendantes - va permettre d'établir une chronologie relativement fine de la pénétration étrangère en pays luba. 


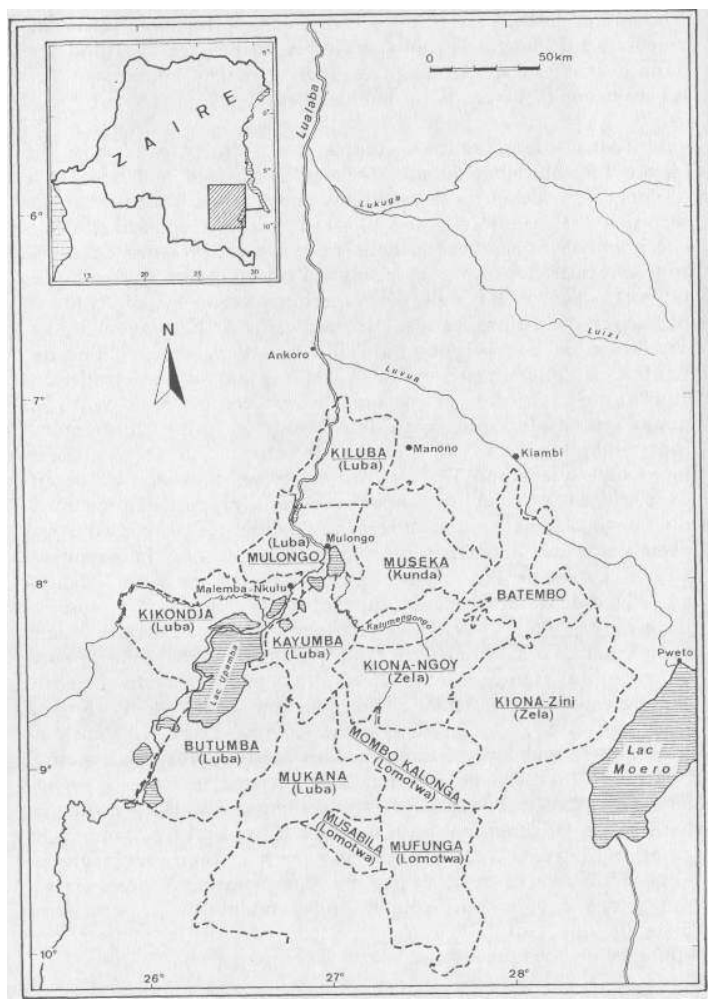

Ainsi, en plus d'un aspect méthodologique non négligeable, l'étude de ces traditions permettra de mieux cerner l'ouverture vers l'extérieur de l'empire luba dans le dernier tiers du $19^{\circ}$ siècle. Tant par l'intérêt méthodologique des sources utilisées que par le regard de l'étranger porté sur les populations africaines, les thèmes abordés dans cette étude illustrent parfaitement un certain nombre de préoccupations chères à Pierre Salmon.

7 Mulongo est une vaste agglomération qui s'étend sur six kilomètres le long de la rive nord du lac Kabamba, à l'exutoire de celui-ci dans le Lualaba. Situé au flanc d'un massif montagneux, Mulongo est localisé à l'extrémité septentrionale de la dépression de l'Upemba et constitue de ce fait même un lieu de passage fréquent d'une rive à l'autre du fleuve Zaïre. Les traditions orales luba font d'ailleurs souvent référence au passage du fleuve en pirogue à cet endroit. Il s'agit donc d'un lieu crucial dans les échanges entre les deux rives du Lualaba. Excentrée par rapport au coeur du pays luba, cette chefferie importante est un point de passage quasi obligé pour tout ceux qui, venant de l'est, désiraient contourner la dépression de l'Upemba, vaste étendue marécageuse, afin de rejoindre la cour royale luba, située plus à l'est, à Kabongo. En plus de cette situation géographique exceptionnelle, il s'agit d'un centre économique important. En bordure du lac Kabamba et du Lualaba, les Luba de Mulongo tirent leur richesse de la pêche et de leur rôle ancestral de passeurs d'eau. C'est aussi un lieu de marché important où convergent des produits venant de la dépression de l'Upemba, du fleuve et des régions montagneuses avoisinantes. Mulongo est donc un lieu stratégique, propice à l'étude de la pénétration étrangère dans le royaume luba au $19^{\circ}$ siècle, d'autant plus qu'une documentation relativement abondante donne de précieux renseignements d'ordre historique sur la région.

8 Lors de son deuxième voyage d'apprentissage, que l'on date de 1855-1860, Tippo Tip écrit: "Nous arrivâmes dans l'Urua, chez le chef Mrongo Tambwe"5. Il y décrit un 
commerce modéré. Il y achète de petites pointes d'ivoire, bien moins chères que les grandes.

Plus tard, lors de son troisième voyage, vers 1870-1882, Tippo Tip parle à nouveau d'un chef Mrongo Tambwe ${ }^{6}$. Après un séjour de plusieurs mois à Kayumba, à l'est de Kinkondja, sur la rive droite du Lualaba (cf. carte), il se rend à Mulongo où il intervient militairement en faveur du chef Mrongo Tambwe évincé par son rival Mrongo Kasanga. Ce récit, plus long et plus précis que le précédent, atteste bien qu'il s'agit là du Mulongo luba, au bord du lac Kabamba. L'itinéraire emprunté par Tippo-Tip et sa description du lac et de ses activités de pêche le confirment. Assimilant logiquement le Mrongo Tambwe $\mathrm{du}$ second voyage d'apprentissage à celui rencontré lors de son troisième voyage, on considère généralement que Tippo Tip aurait donc visité une première fois Mulongo en 1855-1860, avant d'y séjourner plus longuement en 1870-18827.

Cela n'est pas sans importance puisque, dès 1855-1860, Tippo Tip aurait donc atteint Mulongo, c'est-à-dire le pays luba et le fleuve Lualaba à une époque où les autres commerçants arabes se cantonnent encore aux alentours des lacs Tanganyika et Moero. Partant de Mtowa, au nord de l'actuel Kalemie sur les bords du Tanganyika, il aurait traversé tout le nord-Shaba, franchit la Lukuga et la Luvua pour s'arrêter au fleuve Zaïre dans un territoire quasi inconnu, avec un petit groupe constitué d'une vingtaine d'Arabes, lors de la première mission commerciale placée sous sa responsabilité.

Plusieurs éléments du texte de Tippo Tip permettent de douter de la réalité de ce voyage. Partant de Mtowa, il se serait rendu directement à Mulongo avant d'entreprendre presque immédiatement le trajet du retour. Traversant des territoires inconnus, sur une distance de $350 \mathrm{~km}$ à vol d'oiseau, il n'aurait rien eu à raconter de ce périple inédit. De plus, il signale que le commerce est modéré à Mrongo Tambwe. Or, la région du lac Kabamba est riche et prospère comme il le décrit lui-même lors de son troisième voyage . $^{8}$ Enfin, il indique que "tout le monde" achète des grandes pointes d'ivoire. Il est hautement improbable que vers 1855-1860 il y ait tellement d'acheteurs d'ivoire à Mulongo que Tippo Tip puisse écrire que tout le monde en achète. En effet, au début des années 1860, les Arabes se concentrent principalement aux rives sud-ouest du Tanganyika et n'opèrent pratiquement pas d'incursions plus à l'ouest. Par exemple, en 1868, on signale à Livingstone qui séjourne à Kabambare que les Arabes ne sont à cet endroit que depuis fort peu de temps'. Il serait donc étonnant de rencontrer un commerce intense de l'ivoire à Mulongo vers 1855-1860.

12 Malgré cela, Bontinck estime que le Mrongo Tambwe mentionné lors du second voyage d'apprentissage tout comme celui du troisième périple ont été rencontré l'un et l'autre à Mulongo, au bord du lac Kabamba ${ }^{10}$. Pour cela, il va distinguer un Mrongo Tambwe père, rencontré lors du second voyage d'apprentissage d'un Mrongo Tambwe fils qui dispute le pouvoir à son frère Mrongo Kasanga, rencontré lors du troisième voyage. Poursuivant son raisonnement, il rapproche Mrongo Tambwe père du Kilolo Ntambo dont parle Livingstone, tué en 1868 lors d'une guerre contre des Arabes ${ }^{11}$.

13 L'enquête territoriale effectuée en 1909 par l'administrateur Boterdal permet de compléter les données fournies par Tippo Tip et de remettre en question l'arrivée de ce dernier à Mulongo lors de son second voyage. S'intéressant plus particulièrement à l'histoire de la chefferie, cette enquête nous fournit une généalogie complète et commentée des différents Mulopwe de Mulongo. Elle mentionne, à partir d'une récolte des traditions orales sur l'histoire de la région, l'intervention de Tippo Tip dans la lutte qui oppose Mrongo Tambwe à Mrongo Kasanga. Le texte précise même que Tippo Tip y 
reçut le surnom de Mutshiupula que l'on peut rapprocher des surnoms Mutipula ou Mutshipule qui lui sont donnés au Manyema ${ }^{12}$.

On observe une très bonne congruence entre les versions de Tippo Tip et de Boterdal. Parmi ces éléments communs, on relève le trajet emprunté par Tippo Tip pour se rendre de Kayumba à Mulongo et les différents villages traversés; la querelle opposant Mrongo Tambwe à Mrongo Kasanga et l'intervention de Tippo Tip en faveur de ce dernier; la venue d'émissaires de Mrongo Kasanga chez Tippo Tip, chargés de lui vendre de l'ivoire que celui-ci refuse; le vol de casseroles à des membres de son équipage par des gens de Mulongo. Ce vol, du reste, sera le prétexte au déclenchement de la guerre contre Mrongo Kasanga. Enfin, on peut encore citer l'arrivée à Mulongo, peu après le départ de Tippo Tip, de Juma ben Salum Wad Rakad, surnommé Juma Merikani ${ }^{13}$. Ces différents éléments d'ensemble et de détail permettent d'établir qu'il s'agit bien du passage de Tippo Tip à Mulongo lors de son troisième voyage, 1870-1882. Cette extraordinaire corroboration des deux récits montre que le rapport de Boterdal se base vraisemblablement sur des témoins oculaires du passage de Tippo Tip, ce qui est tout à fait possible puisque la rédaction de ce rapport a eu lieu au maximum trente ans après les faits.

enors de cet épisode particulier, l'enquête établit l'historique de Mulongo, depuis l'accession au pouvoir de son premier chef, intronisé par le Roi luba Kumimbwe Ngombe, dont le règne s'étend de 1810 à $1840^{14}$. Dès la création de cet Etat, on assiste à une lutte, classique en pays luba, entre deux lignages royaux en vue de s'approprier le pouvoir. Cette histoire, fort détaillée et corroborée par d'autres généalogies de Mulongo, ne parle ni d'un Mulongo Tambwe père, ni d'un précédent voyage de Tippo Tip dans la région ${ }^{15}$. Or, vu la précision de la généalogie fournie et les nombreux détails restitués suite au passage de Tippo Tip lors de son troisième voyage, s'il y avait séjourner précédemment, les traditions récoltées par Boterdal le mentionneraient très vraisemblablement.

Par contre, ce rapport apporte la preuve du passage de Tippo Tip à Mulongo lors de son troisième voyage. Ce séjour dut avoir lieu au cours des années 1871/1872. En effet, en août 1874, Cameron rencontre Tippo Tip qui lui précise qu'il a séjourné au Katanga depuis presque 2 ans ${ }^{16}$. Or, peu de temps avant sa rencontre avec Cameron, il nous renseigne de "la troisième année de mon séjour dans l'Utetera" ${ }^{17}$. On peut donc en déduire qu'il arrive en pays Tetela au début ou courant de l'année 1872, après avoir quitté Mulongo. Ainsi, son séjour de 9 mois à Mulongo doit avoir eu lieu au cours des années 1871/72 et son séjour d'un an à Kayumba en 1870/71 ${ }^{18}$. Un témoignage de Livingstone confirme ces dates puisqu'en juin 1872, depuis Tabora, il apprend les informations suivantes : "Tippo Tip ... at Katanga and had purchased much ivory from Kayombe or Kayombo in Rua"19. Cette information atteste bien de sa présence à Kayumba pour la période considérée.

17 Pour se rendre à Kayumba, petit Etat luba client de la cour centrale, Tippo Tip part de la rive orientale du lac Moero, passe par Mpweto en pays tabwa et traverse les villages lomotwa dans les monts Kundelungu (cf. carte), soit une distance de $350 \mathrm{~km}$ à vol d'oiseau. ${ }^{20}$ Auparavant, il s'était rendu en pays bemba. A. Roberts confirme ce passage chez les Bemba et le date de 1869/70. Ces différentes informations correspondent donc avec son départ de Bagamoyo en Tanzanie, aux environs de 1869 et non en 1870/71, comme on le pense généralement ${ }^{21}$.

18 Cependant, un événement décisif, raconté par Tippo Tip et concernant la mort de Kazembe VII Muongo pose problème à l'établissement d'une telle chronologie ${ }^{22}$. Tous les indices historiques laissent supposer que ce dernier a été tué en $1872^{23}$. Dans ce cas, il est matériellement impossible que Tippo Tip, qui séjourne alors à Mulongo, ait participé à ce 
meurtre comme il l'affirme lui-même. En accord avec Roberts, nous pensons qu'en fait Tippo Tip n'a pas pris part à la guerre opposant Kazembe VII à des marchands arabes. Dans ses souvenirs, il se serait attribué à tort cette exécution par contamination avec différentes traditions arabes concernant la région. Il est étonnant de constater que dans la Maisha, la guerre contre Kazembe VII se résume en 5 lignes vagues et imprécises alors que la guerre contre Mulongo Kasanga, chef de bien moindre importance, y est racontée en 6 pages avec force détails. Notre propos n'étant pas d'analyser plus avant cet épisode, nous renvoyons à l'argumentation fort convaincante de Roberts ${ }^{24}$.

Un autre élément permet de mettre en doute la réalité de ce premier séjour à Mulongo. En 1871/72, Tippo Tip ne fait aucune allusion au fait qu'il serait passé par là antérieurement, mais, par contre, il décrit longuement le pays, ses activités économiques et les guerres qu'il y mène. Ces différents indices nous permettent d'affirmer que ce n'est pas à Mulongo, en bordure du lac Kabamba que s'est rendu Tippo Tip lors de son second voyage d'apprentissage. Il nous faut dès lors tenter d'identifier le Mrongo Tambwe qu'il prétend avoir rencontré à cette occasion, afin de découvrir qu'elle était la région parcourue à cette époque par Tippo Tip.

Tout d'abord, ce personnage ne peut être le père du Mrongo Tambwe rencontré en 1871/72. Pour étayer cette hypothèse, Bontinck ${ }^{25}$ se réfère à un passage de Tippo Tip expliquant que Mrongo Tambwe et Mrongo Kasanga seraient des frères (ndugu), se disputant la chefferie de leur père (baba). Cependant, aucune des généalogies de Mulongo ne fait référence à un Mrongo Tambwe père. Par contre, la généalogie des chefs fournie par Boterdal permet de mieux comprendre les propos de Tippo Tip ${ }^{26}$. En effet, s'ils appartiennent à des lignages rivaux se disputant le pouvoir, Mulongo Tambwe (du lignage Musumba) et Mulongo Kasanga (du lignage Membe) sont apparentés et descendent du premier Mulopwe de Mulongo, Mulongo Kalala Panda, investi chef sous le règne du roi Luba Kumwimba Ngombe, 1810-184027. La généalogie de Mulongo confirme donc en partie les informations de Tippo Tip: il s'agit de deux parents de même génération qui se disputent la chefferie de leur ancêtre. Aucun élément historique ne permet cependant d'affirmer qu'il s'agit de deux frères réclamant le pouvoir de leur père biologique.

21 Par ailleurs, Bontinck identifie ce premier Mrongo Tambwe à un personnage mystérieux dont parle Livingstone: Kilolo Ntambo ${ }^{28}$. A la fin du mois d'octobre 1868, aux environs de Mpweto, le célèbre explorateur apprend, de l'Arabe Saïd ben Habib, le meurtre de son frère, Salem ben Habib. Il aurait été tué par les Bakatala dans l'ouest Lualaba car Sef, un autre commerçant arabe, avait ravagé la région et attaqué Kilolo Ntambo. En représailles, Said ben Habib fit la guerre dans la région et amassa un butin considérable avant de s'en retourner définitivement à Zanzibar. "Ce sont les Bakatala, riverains du Loualaba occidental qui ont tué Salem ben Habib. S'éloigner d'eux. Makouammba est l'un des chefs des habitants des cavernes. Ngoulou aussi ; Masika-Kitoboué est chef des Balouba. Sef s'est 'attaché' Kilolo N'tammboué".

22 Avant d'établir un tel rapprochement avec Mrongo Tambwe, il est nécessaire de tout d'abord identifier le Kilolo Ntambo de Livingstone. Il faut savoir que "Kilolo" est un titre luba désignant un notable : chef de terre ou de village, chef de guerre ou dignitaire de la cour du Mulopwe ${ }^{29}$. Il s'agit d'un terme à la signification flottante, qui ne s'attache pas à une fonction politique spécifique. On le retrouve d'ailleurs chez toutes les populations parlant une langue de la zone L : Sanga, Kaonde, Zela, Lomotwa, Nwenshi etc... préalablement les différents protagonistes de cette histoire de vengeance. 

Ntambo dans cette même région puisque son rôle est lié aux actions des frères ben Habib
et de Sef. Dans un tel contexte, il est possible d'identifier cinq personnages répondant à
l'ébauche de description fournie, pouvant porter le titre de Kilolo et le nom de Ntambo. 1. Il existe un village Tambo sur la rivière Kalumengogo, à la frontière des chefferies zela de Kiona Ngoy et Museka. Le titre de chef de village, dans cette région, étant Kilolo, le chef de Tambo devait, selon toute vraisemblance, s'appeler Kilolo Ntambo.

30 2. Au sud-est des Zela se trouvent les populations Batembo. Durant toute la période coloniale, les administrateurs belges vont confondre Zela et Batembo, au point de n'en faire qu'une seule et même population. Il est donc possible que Kilolo Ntambo soit en fait un chef Tembo ${ }^{37}$.

31 3. Selon le père Stocky, qui a travaillé à Museka et à Kayumba, au sud, dans les monts Kibara, se trouvait au moment de l'arrivée des Européens dans la région, le domaine du chef Tumbe, lequel se disait Luba ${ }^{38}$. Ce personnage pourrait très bien être identifier à Kilolo Ntambo. 
32 4. Un des quatre lignages royaux pouvant revendiquer légitimement le titre de Mulopwe chez les Zela de Kiona Ngoy est le lignage Ntamba. Or, comme nous l'avons fait remarquer précédemment, le chef de lignage ou de village chez les Zela porte le titre de Kilolo ${ }^{39}$.

5. Le chef de guerre, le Mwadya Mvita des Lomotwa du chef Mufunga est appelé Kilolo $\mathrm{Ntambo}^{40}$.

Ces différentes identifications possibles renforcent notre hypothèse que les représailles de Saïd ben Habib ont bien eu pour cadre le pays zela ou chez leurs voisins, les Lomotwa ou les Tembo. En effet, dans cette région, pas moins de cinq personnages dont la fonction a toujours un rapport direct avec le pouvoir ou avec la guerre peuvent prétendre au titre de Kilolo Ntambo. Malheureusement, le manque de sources sur cette région qui a peu retenu l'attention des historiens et des ethnologues ne permet pas de préciser d'avantage l'identité de ce personnage. Tout au plus pouvons nous dire que Kilolo Ntambo était un dignitaire politique de cette région entre Moero et Lualaba.

Cependant, ces éléments ne permettent pas d'affirmer que le Mrongo Tambwe rencontré par Tippo Tip lors de son second voyage d'apprentissage ait un lien quelconque avec le Kilolo Ntambo de Livingstone. Un seul indice, et des plus ténu, donne une certaine validité au rapprochement de Mrongo Tambwe avec Kilolo Ntambo : Tippo Tip indique la présence d'un certain commerce avec les Arabes chez Mrongo Tambwe. Et, en effet, il semble bien que dès la fin des années 1860 , des commerçants arabes, comme les frères ben Habib et Sef, sillonnaient le pays des Zela et des Lomotwa. Il se peut donc que Tippo Tip ait en fait parcouru cette région lors de son second voyage d'apprentissage.

Pour aller plus avant dans l'identification de Kilolo Ntambo, et par là même de Mrongo Tambwe, il faut encore préciser que l'on a régulièrement attribué le meurtre de ce chef à Sef, épaulé d'alliés yeke de $\mathrm{M}^{\prime} \mathrm{siri}^{41}$. Il s'agit à nouveau d'une confusion, reposant simplement sur des similarités onomastiques, à savoir ce fameux titre de Kilolo Ntambo.

Cette confusion prend sa source dans un récit de l'administrateur Verdick qui décrit à sa manière la mort de Salem ben Habib ${ }^{42}$. Selon lui, un Arabisé nommé Saïdi voulut se rendre au Lualaba et fut attaqué par un chef luba, Kilolo N'Tambwe. Au cours de ce combat, son jeune frère fut tué (Livingstone parle de son frère aîné). Saïdi demande alors à M'siri des guerriers afin de venger la mort de son frère. Il envahit le pays de Kilolo N'Tambwe et tue ce dernier. Par après, fortune faite, il rentre avec éclat à Zanzibar.

En analysant attentivement le texte de Verdick, on se rend compte qu'il a opéré la fusion de deux histoires distinctes. D'une part, il s'est inspiré du récit de Livingstone concernant Saïd ben Habib et Kilolo Ntambo, comme le montrent différentes contaminations : Salem, le frère aîné de Saïd chez Livingstone devient un frère cadet chez Verdick; le retour de Saïd à Zanzibar est nettement copié de Livingstone, aucune tradition du Shaba ne pouvant, géographiquement, incorporer cet élément. D'autre part, il a mélangé ce récit à une tradition historique yeke, où Nkala, un chef Sanga, va demander l'aide militaire de M'siri suite à des raids luba. Ces Luba, avec à leur tête un certain Kilolo Ntambo, furent vaincus par les Yeke à la rivière Kabangu, près de Mokabe Kazari, au nord de Bunkeya, soit au coeur du pays Sanga ${ }^{43}$. En fait, il s'agit là d'un tout autre événement qui se situe au début de l'implantation yeke au Shaba, bien avant 1868 et, de plus, localisé en pays Sanga, à quelque $400 \mathrm{Km}$ au sud du territoire zela.

39 Bontinck, pour sa part, estime ce rapprochement possible car les Yeke affirment avoir été aidé, dans cette campagne, par un Swahili du nom de Nsefu, qu'il rapproche du Sef cité par Livingstone ${ }^{44}$. Cependant, ce Nsefu n'est jamais cité pour sa participation à cette 
guerre par les traditions yeke, mais pour sa découverte de l'or de Kambove ${ }^{45}$. Quant à Saïdi, que Verdick fait participé au même épisode, aucune tradition ne mentionne sa présence. Si l'histoire yeke parle bien, pour d'autres événements, d'un certain Saïdi (un village portait ce nom, au nord de la capitale Yeke, Bunkeya), aucun élément ne permet d'affirmer qu'il s'agit bien de Saïd ben Habib. Bien au contraire, Tippo Tip signale qu'un Arabe nommé Saïd ben Ali, compagnon de route lors de son séjour à Mulongo en 1871/72, l'a quitté afin de suivre des émissaires de M'siri et de commercer en pays yeke ${ }^{46}$. Le Saïdi de la tradition yeke s'identifie plutôt à ce personnage qui séjourna longuement à Bunkeya.

40 Le texte de Verdick est donc le résultat d'une fusion de deux épisodes historiques distincts, sur base, semble-t-il, de l'homonymie de deux personnages appelés chacun Kilolo Ntambo. Cependant, il est aisé d'identifier le Kilolo Ntambo de l'épisode yeke. Il était chef du village Tambo, situé à quelques kilomètres au nord de Mokabe Kazari et, donc, sans aucun rapport avec son homonyme cité par Livingstone.

41 Cette analyse approfondie de différentes sources concernant l'histoire du Shaba dans la seconde moitié du $19^{\circ}$ siècle nous permet de préciser tant la chronologie des événements que ses principaux acteurs et ainsi de mieux comprendre les informations fournies par Tippo Tip et Livingstone. Tout d'abord, il est certain que le Mrongo Tambwe rencontré par Tippo Tip lors de son second voyage d'apprentissage n'est pas Mulongo Tambwe, Mulopwe de Mulongo et, d'autre part, que Tippo Tip ne s'est pas rendu à Mulongo lors de ce voyage.

Mais, dans ce cas, où s'est-il rendu ? Pour répondre à cette question, on se rappellera qu'il est nécessaire de localiser la région où il se serait rendu et de rapprocher Mrongo Tambwe du Kilolo Ntambo de Livingstone. Dans ce cadre, on a pu déterminer que la campagne menée par Saïd ben Habib pour venger la mort de son frère, et où intervient ce fameux Kilolo Ntambo, s'est déroulée en pays zela ou dans une contrée limitrophe, chez les Lomotwa ou les Batembo, dans une région fort éloignée de Mulongo. Enfin, la guerre menée par les Yeke, aidés de Swahili, contre un certain Kilolo Ntambo n'a aucun rapport avec l'épisode de Saïd ben Habib puisqu'elle a eu lieu au moins 30 ans auparavant, dans une région différente. On ne peut donc identifier ce personnage de la tradition yeke à celui dont parlait Livingstone.

43 A partir de ces différents éléments, il est possible à présent de tenter d'identifier le personnage rencontré par Tippo Tip lors de son voyage de 1855-1860.

44 Soit Mrongo Tambwe serait bien le Kilolo Ntambo de Livingstone, et donc, dès 1855/60, soit bien avant Saïd ben Habib et son frère, Tippo Tip aurait fait une incursion en pays zela ou dans les environs.

Soit Tippo Tip n'aurait jamais rencontré de personne de ce nom lors de son second voyage d'apprentissage et aurait subi une contamination due aux aventures de Saïd ben Habib. Il aurait appelé ce personnage Mrongo Tambwe par confusion avec le chef luba réellement rencontré en 1871/72; ces deux individus s'appelant chacun Tambwe. Cette hypothèse est plausible puisque Tippo Tip écrit ses mémoires quarante ans après le déroulement de ces événements et sans notes. D'autres contaminations du même ordre émaillent son récit. Ainsi, par exemple, il s'est attribué le meurtre de Kazembe VII Muongo sous influence d'autres traditions arabes concernant la région du Luapula. 
Non loin de le rive occidentale du Tanganyika et de Mtowa où débarqua Tippo Tip, plusieurs villages portent ce nom :

- Tambwe à l'est de Kongolo

- Tambwe à l'ouest de Kabambare

- Mulongo entre Kabalo et, plus à l'est, Nyunzu.

Il est plausible qu'il ait séjourné dans l'un de ces villages car, dès cette époque, un commerce avec les Arabes s'y développait. Cette solution a l'avantage de conforter la version de Tippo Tip qui fait allusion à une certaine activité commerciale dans la contrée visitée. De plus, comme il indique n'avoir fait qu'un aller-retour entre ce village et Mtowa, cette hypothèse est, géographiquement, la plus plausible. Dans ce cas, ce serait aussi par contamination avec son voyage suivant à Mulongo, au bord du lac Kabamba, qu'il aurait appelé le chef de ce village Mrongo Tambwe.

Sans avoir un rapport direct avec le problème qui vient de nous occuper, les traditions de Mulongo nous donnent d'autres informations intéressantes concernant la pénétration arabe dans la région. En effet, tant la Maisha de Tippo Tip que les traditions récoltées par Boterdal nous informent du passage à Mulongo d'un autre commerçant arabe, Juma Merikani. La complémentarité des deux sources autorisent une analyse approfondie de ce séjour qui, de par ses objectifs et son déroulement, semble avoir été calqué sur celui de Tippo Tip.

En 1872, peu de temps après avoir quitté Mulongo, Tippo Tip rencontre Juma ben Salum wad Rakad, - surnommé Juma Merikani à cause des tissus américains qu'il commerçait ${ }^{47}$ dans la chefferie Kiluba, au nord de Mulongo, le long du Lualaba (cf. carte) ${ }^{48}$. D'après Cameron, qui rencontra Juma Merikani en octobre 1894, ce dernier avait fondé un établissement permanent dans cet état tributaire de la court luba ${ }^{49}$. Grâce au témoignage de Tippo Tip qui précise que c'est la première fois que Juma Merikani sillonne cette région, on peut dater la fondation du camp permanent dans la chefferie Kiluba de $1872 / 73$.

50 Les traditions de Mulongo, quant à elles, indiquent qu'après le départ de Tippo Tip, un autre Arabe, Fwamba Melikoni, que l'on peut identifier sans erreur à Juma Merikani, s'est installé à son tour à Mulongo. Se mêlant aux affaires politiques du Royaume, il en vient à chasser le chef Mulongo Tambwe et rappelle son rival Mulongo Kasanga ${ }^{50}$. Ces événements durent aussi se dérouler au cours des années 1872/73.

51 Durant son séjour à Kiluba, en plus de son incursion à Mulongo, Merikani a effectué plusieurs voyages plus au sud, en territoire yeke où, d'après Cameron, il visita des mines d'or et de cuivre, à Kambove notamment. Par la suite, en octobre 1874, il installe son établissement au coeur du royaume luba, à proximité de la résidence de Kasongo Kalombo ${ }^{51}$.

Grâce aux témoignages de Cameron et de Tippo Tip, nous pouvons désormais établir avec certitude que Juma Merikani s'est installé à Kiluba de 1872/73 à 1874. A partir de ce camp, il sillonna tout le Shaba, Mulongo et les régions cuprifères sous contrôle Yeke, avant de s'établir plus au nord, à Kilumba, village avoisinant la résidence de Kasongo Kalombo.

C'est à nouveau grâce aux traditions recueillies à Mulongo au début de notre siècle qu'il est possible de retracer les voyages de Juma Merikani et de leur donner un cadre chronologique cohérent. Ce que la rencontre avec Tippo Tip nous laissait supposer, à savoir un passage de Merikani par Mulongo, nous est bien confirmé par les témoignages recueillis à Mulongo par Boterdal. 
54 étudiée ici, a permis de mettre en évidence la bonne congruence des traditions orales de Mulongo, rapportées par Boterdal en 1909, et les souvenirs que Tippo Tip avait gardé de son troisième voyage. Ainsi corroborée, la valeur historique de ces sources nous apparait non seulement dans ces aspects historiques mais aussi pour les informations ethnographiques qu'elles contiennent. En effet, celles-ci ne se contentent pas de nous informer sur des éléments historiques, d'ordre généalogique ou politique. Elles permettent aussi d'appréhender des événements plus quotidiens, plus particuliers, comme le type de vaisselle employé par les commerçants arabes ou la façon dont les populations ressentent ou exploitent, à des fins politiques ou de prestige, la présence d'étrangers. En effet, la venue de commerçants n'apporte pas seulement un accroissement des biens matériels, lié à l'extension du commerce mais, à un niveau plus symbolique, elle permet de rehausser le prestige des chefs de ces régions. Ainsi, par exemple, avant de se rendre à Mulongo, Tippo Tip séjourna durant plusieurs mois à Kayumba. Plutôt qu'à une installation liée au commerce, on a l'impression que ce séjour s'apparente beaucoup plus à une opération de relation publique. Tippo Tip y reçoit les émissaires de nombreux chefs tels Museka, Mulongo Tambwe ou M'siri qui, tous, l'invitent à séjourner chez eux ${ }^{52}$. En plus d'objectifs commerciaux, ces invitations répondent à une stratégie de compétition, liée au prestige politique d'une telle présence. Enfin, le passage des Arabes étaient aussi l'occasion de conforter ou de rétablir certaines positions politiques. C'est Mulongo Tambwe lui-même qui invite Tippo Tip. L'objectif principal de celui-ci n'est pas tant de commercer avec ce dernier, mais d'obtenir son appui afin d'évincer les prétendants du lignage royal rival et d'ainsi accéder au pouvoir, avec d'autant plus de facilité que son prestige était rehaussé par cette présence. Ce sont les mêmes motivations qui pousseront ensuite Mulongo Kasanga à faire appel à Juma Merikani pour retrouver la fonction de Mulopwe que lui avait ravi Tambwe avec l'aide de Tippo Tip. l'accueil des commerçants arabes : ils amèneront - croit-on -, un accroissement des biens matériels, du prestige et conforteront le pouvoir du chef. Il est significatif de constater que ce sont presque les mêmes motivations - l'économie et une main-mise politique sur la région - qui sont le moteur des activités des Arabes. Au départ, c'est donc sur base d'une communauté d'intérêts que vont s'établir les relations commerciales entre traitants arabes et populations autochtones, chacun élaborant des stratégies en fonction d'objectifs finalement assez similaires. Il faut donc se départir d'une vue caricaturale véhiculée par la propagande anti-esclavagiste et se rendre compte de la subtilité des rapports qui se sont noués durant cette période entre les commerçants swahili et les chefs locaux.

Ces récits permettent aussi de mieux appréhender la manière dont les commerçants arabes pratiquaient leur commerce et s'ingéraient dans les affaires politiques des populations rencontrées. Juma Merikani, tout comme Tippo Tip, dès son arrivée à Mulongo, prend parti dans les querelles politiques locales et place à la tête de la chefferie un chef qui lui est tout dévoué puisqu'il lui doit son accession au pouvoir. Il ne s'agit cependant pas, pour lui, d'exercer une réelle domination politique à long terme sur la région, mais de profiter des querelles de succession existantes et de la rivalité des lignages royaux pour placer un chef qui lui sera dorénavant redevable. Contrairement aux Yeke, l'objectif des traitants arabes n'est pas une occupation durable du pays mais son exploitation économique maximale dans un laps de temps relativement court. Les 
Yeke, en arrivant à Mulongo, pratiqueront une politique en apparence similaire à celle des Arabes, à la différence près qu'ils visent une installation à long terme et une occupation politique effective de la région. Ces deux éléments aboutiront rapidement à un changement radical dans le mode d'exploitation commerciale et de domination des populations autochtones qui rendra la présence yeke beaucoup plus fondamentale dans la vie politique et économique de Mulongo ${ }^{53}$.

Dans un tout autre ordre d'idée, il faut aussi mettre en évidence la prodigieuse mémoire de Tippo Tip et la valeur historique de sa Maisha. Pour son séjour à Mulongo en 1871/72, les noms et les détails donnés sont tous corroborés par la tradition locale. Ses souvenirs sont nombreux et précis puisque, quarante années plus tard, il nous restitue ces événements en plus de six pages. Mais cette stupéfiante mémoire a son revers. En effet, pour d'autres épisodes, il commet différentes confusions entre sa propre histoire et les traditions d'autres Arabes de la région. Il en va ainsi de son hypothétique séjour à Mulongo en 1855/60 ou du meurtre de Kazembe VII Muongo. Remarquons que dans les deux exemples cités, le récit est bref et vague : il s'agit à chaque fois de quelques lignes sans détails, "glissées" entre des événements racontés de façon plus précise, comme s'il doutait de sa mémoire.

Ainsi, la structure formelle de la Maisha parait fort contrastée. Elle se compose d'une part de passages longs et détaillés, où l'on voit apparaître un Tippo Tip sûr de lui, de sa mémoire. En général, ces longs épisodes peuvent être validés par d'autres sources, comme c'est le cas ici. Mais, à d'autres moments, nous avons affaire à un homme concis et vague, qui rapporte des événements que d'autres sources ignorent ou contredisent. Il semble trahi par ses souvenirs sous l'effet de contaminations ou de confusions dues aux contacts des récits d'autres traitants arabes.

Au-delà de ces deux conclusions qui sont avant tout d'ordre méthodologique et porte sur la validité historique des sources orales africaines et arabes, l'analyse et la confrontation des souvenirs de Tippo Tip et des traditions de Mulongo permettent d'établir une chronologie relativement sûre et précise du début du troisième voyage de Tippo Tip et des événements politiques relatifs à la chefferie de Mulongo aux alentours de 1870. Ceci confirme enfin que cette région est restée en dehors de la sphère d'influence directe des Arabes jusqu'au dernier quart du $19^{\circ}$ siècle.

\section{BIBLIOGRAPHIE}

\section{A. Ouvrages non publiés.}

BOTERDAL, Rapport d'enquête. Chefferie de Mulongo, PV n¹2, 14/12/1909.

A. BOULANGER, Recherche sur la société et la religion Zela, Paris, thèse de $3^{\circ}$ cycle, EPHE, $5^{\circ}$ sect., 1978. 
N.J. FERBER, Enquête sur les Museka, 30/10/1930, Archives Sous Région du Haut Lomami (SRHL), microfilms Reefe.

HEUTSEBAUT, Chefferie de Mulongo, historique, 30/10/1918, archives SRHL.

Historique des successions de chefs dans la chefferie de Mulongo, 1/10/1909, archives SRHL.

J. HOOVER, The Seduction of Ruzwej, PhD, 1978.

LONGFILS, Chefferie Museka. Généalogie, 30/6/1926, archives SRHL.

S.A. LUCAS, Baluba et Aruund, Paris, thèse de $3^{\circ}$ cycle, 1968.

H. LEGROS, Enquêtes de terrain à Bunkeya, du 1/2/1991 au 8/4/1991.

TERVUEREN, Dossier Père Stoky, MRAC, archives de la section Préhistoire, PR.178.

\section{B. Ouvrages publiés.}

F.S. ARNOT, Garenganze or seven years' pioneer work in Central Africa, Londres, 1889.

a. F. BONTINCK, L'autobiographie de Hamed ben Mohammed el Murjebi Tippo Tip (ca 1840-1905), ARSOM, NS XLII-4, Bruxelles, 1974.

b. F. BONTINCK, La double traversée de l'Afrique par trois "Arabes" de Zanzibar, 1845-1860, in Etudes d'Histoire Africaine, VI, 1974, p. 3-50.

W.F.P. BURTON, Luba Religion and Magic in Custom and Belief, Tervueren, 1961.

V.L. CAMERON, Across Africa, 2 vol., Londres 1877 ; trad, fr., H. LOREAU, A travers l'Afrique, Paris, 1881.

H. CAPELLO \& R. IVENS, De Angola a Contra-Costa, vol. 2, Lisbonnes, 1886.

I. CUNNISON, The Luapula Peoples of Northern Rhodesia, Manchester, 1959.

G. DE PLAEN, Diplomatie et économie. Le système Yeke, in Cultures et Développement, 1979, XI, 1, p. 3-46.

D. LIVINGSTONE, LastsJournals, ed. by H. WALLER, 2 vol., Londres, 1873 ; trad. fr. H. LOREAU, Dernier Journal du Docteur D. Livingstone, 2vol., Paris, 1876.

Mémoires de Mukanda Bantu, in Bulletin de la Société Belge d'Etudes Coloniales, 1919, n 5, p. 251-277, nº, p. 497-521.

A. MUNONGO, Mort de la Mugoli (reine) Mahanga, in Bulletin du CEPSI, 1951, 17, p. 260-263.

A. MUNONGO, Court aperçu de l'histoire yeke, in Bulletin du CEPSI, 1967, 25, p. 7-20.

P. REICHARD, Bericht über die Reise nach Urua und Katanga, in Mitteilungen der Afrikanischen Gesellschaft in Deutschland,1885, IV, 5, p. 303-309.

F. RENAULT, Tippo Tip. Un potentat arabe en Afrique Centrale au XIXº siècle, Paris, 1987.

A.D. ROBERTS, Tippu Tip, Livingstone and the Chronology of Kazembe, in Azania, 1967, 2, p. 115-131.

A.D. ROBERTS, A History of the Bemba, Londres, 1973.

O.F.M. VAN AVERMAET, Dictionnaire Kiluba-Français, Tervueren, 1954.

W. VAN DORPE, Origine et migration des Yeke, Bandundu, Ceeba série 2, vol. 43, 1978.

J. VANSINA, Kingdoms of the Savanna, Madison, 1966. 
J. VANSINA, Oral Tradition as History, Madison, 1985.

A. VERBEKEN, Msiri, roi du Garenganze, Bruxelles, 1956.

E. VERDICK, Les premiers jours au Katanga, Bruxelles, 1952

E. VERHULPEN, Baluba et Balubaïsés du Katanga, Anvers-Paris, 1936.

W.H. WHITELEY, Maisha ya Hamed bin Muhammed el Murjebi yaani Tippu Tip, East African Literature Bureau, 1959 (1974).

A. WILSON, Long distance trade and the Luba Lomami Empire, in Journal of African History, 1972, XIII, 4, p. 575-589.

\section{NOTES}

1. Pour de plus amples informations sur l'histoire Luba, cf. T.Q. Reefe, 1981.

2. J. VANSINA, 1985, pp. 158-160.

3. Cf. F. BONTINCK, 1974, pp. 6-10.

4. Ibidem, p. 190.

5. F. BONTINCK, 1974, § 4, p. 42.

6. Ibidem, § 72-82, pp. 78-94.

7. F. BONTINCK, 1974, n. 18, pp. 182-183 ; G. DE PLAEN, 1979, pp. 15-18; T.Q. REEFE, 1981, pp. 164-165 ; J. VANSINA, 1965, p. 236.

8. F. BONTINCK, 1974, § 75, pp. 80-81.

9. D. LIVINGSTONE, 1873, II, p. 58 ; A. WILSON, 1972, pp. 581-582.

10. F. BONTINCK, 1974, n. 18, pp. 182-183.

11. D. LIVINGSTONE, 1873, I, p. 335.

12. BOTERDAL, 1909, p. 3 ; F. BONTINCK, 1974, n. 95, p. 200 ; F. RENAULT, 1989, p. 230.

13. F. BONTINCK, 1974, § 74-81, pp. 80-83 ; BOTERDAL, 1909, pp. 3-4.

14. BOTERDAL, 1909, pp. 1-2 ; T.Q. REEFE, 1981, pp. 129-134.

15. W.F.P. BURTON, 1961, pp.16-18; HEUTSEBAUT, 1918, pp.1-4; E. VERHULPEN, 1936, pp. 371-372.

16. V.L. CAMERON, 1877, II, p. 12.

17. F. BONTINCK, 1974, § 102, p. 96

18. Ibidem, § 72, p. 79, § 82, p. 84.

19. D. LIVINGSTONE, 1873, II, p. 194.

20. F. BONTINCK, 1974, § 69-70, p. 77.

21. A.D. ROBERTS, 1973, pp. 136-195.

22. F. BONTINCK, 1974, § 68-69, pp. 76-77.

23. F. RENAULT, 1987, pp. 54-55 ; A.D. ROBERTS, 1967, pp. 118-121 ; 1973, p. 199.

24. A.D. ROBERTS, 1967 , pp. 118-121.

25. F. BONTINCK, 1974, n. 18, p. 182.

26. Ibidem, § 74, p. 80.

27. BOTERDAL, 1919, pp. 1-4 ; T.Q. REEFE, 1981, p. 134sq ; E. VERHULPEN, 1936, pp. 371-373.

28. F. BONTINCK, 1974, n. 18, pp. 182-183 ; D. LIVINGSTONE, 1873, I, p. 335 ; II, p. 19.

29. O.F.M. VAN AVEMAET, 1954, p. 360.

30. F. BONTINCK, 1974b, pp. 3-50.

31. D. LIVINGSTONE, 1873, I, pp. 336-337.

32. D. LIVINGSTONE, 1873, I, p. 337 ; A.D. ROBERTS, 1967, pp. 118-121.

33. E. VERDICK, 1951, p. 92 ; E. VERHULPEN, 1936, p. 383. 
34. D. LIVINGSTONE, 1873, II, p. 19.

35. V.L. CAMERON, 1877, II, pp. 89-90, 314

36. F.S. ARNOT, 1889, p. 198 ; A. VAN MALDEREN, 1936, p. 174 ; T.Q. REEFE, 1981, pp. $140-141$.

37. E. VERHULPEN, 1936, p. 56, 139, 382-383.

38. Lettre de Stocky à Bequart, 1942, Tervueren, archives section préhistoire, dossier PR 278

39. A. BOULANGER, 1977 , p. 51

40. A. VAN MALDEREN, 1936, p. 176

41. F. BONTINCK, 1974, n. 18, pp. 182-183.

42. E. VERDICK, 1951, pp. 37-38.

43. Mémoires de Mukanda Bantu, 1919, pp. 254-255 ; A. MUNONGO, 1967a, p. 12 ; Mwanangwa Nsamba Muloji \& Mwanangwa Kafuku, 27 mars 1991, Bunkeya.

44. F. BONTINCK, 1974, n. 18, p. 183.

45. Mémoires de Mukanda Bantu, 1919, p. 260.

46. F. BONTINCK, 1974, § 83, p. 84 .

47. Ces tissus assez grossiers de toile blanche sont d'ailleurs toujours vendus au shaba et dénommés "melikani".

48. F. BONTINCK, $1974, \S 84$, pp. 84-85.

49. V.L. CAMERON, 1877, II, pp. 51-56 ; Cf. F. BONTINCK, 1974, n. 230-231; T.Q. REEFE, 1981, p. 164.

50. BOTERDAL, 1909 , p. 5.

51. V.L. CAMERON, 1877, II, pp. 51-56.

52. F. BONTINCK, 1974, § 72-74, pp. 78-80.

53. Une prochaine publication aura pour thème cette occupation de Mulongo par les Yeke.

\section{RÉSUMÉS}

The tribal lands of the Luba of Mulongo, situated at the extreme north of the Upemba depression are not only an almost obligatory point of passage for those coming from the east en route to the heart of the Luba but are also an important market. It is therefore a very useful place to study the Arab-Swahili penetration in the kingdom of Luba in the nineteenth century, especially as one of the first merchants to reach this chefferie was none other than Tippo Tip. In his autobiography, he briefly speaks of his incursions in Luba but up to now, no other source has permitted the verification or the completion of the information provided by Tippo Tip.

An unpublished administrative report dating from 1909, written up from the oral tradition of the precedent century, tells of power struggles between two rival lineages of the Mulongo royalty. The report not only provides a complete ancestry of the chiefs but also allows the accurate dating of the stages of Arab-Swahili penetration in the kingdom of Luba. Furthermore, this document refers to the passage of Tippo Tip in the region and gives us much information regarding the events that took place during his stay. The congruence of the sources enables a relatively detailed chronology of the passage of Tippo Tip to be established and a better definition of the forms of the penetration and the political domination of Arab-Swahili at the heart of the Luba empire. 


\section{AUTEURS}

\section{PIERRE DE MARET}

Faculté de Philosophie et Lettre - Université Libre de Bruxelles - Belgique

\section{HUGUES LEGROS}

Fonds National Belge de la Recherche Scientifique - Université Libre de Bruxelles - Belgique 